\title{
La narration au service de la politique monétaire : étude comparée du premier discours à Mansion House de deux gouverneurs de la Banque d'Angleterre
}

Narration in the service of monetary policy: a comparative study of the maiden Mansion House speeches by two Governors of the Bank of England

\section{Laurence Harris}

\section{(2) OpenEdition}

Journals

Édition électronique

URL : http://journals.openedition.org/asp/4881

DOI : 10.4000/asp.4881

ISSN : 2108-6354

\section{Éditeur}

Groupe d'étude et de recherche en anglais de spécialité

\section{Édition imprimée}

Date de publication : 1 novembre 2016

Pagination : 49-64

ISSN : 1246-8185

\section{Référence électronique}

Laurence Harris, « La narration au service de la politique monétaire : étude comparée du premier discours à Mansion House de deux gouverneurs de la Banque d'Angleterre », ASp [En ligne], 70 | 2016, mis en ligne le 01 novembre 2017, consulté le 01 novembre 2020. URL : http:// journals.openedition.org/asp/4881; DOI : https://doi.org/10.4000/asp.4881

Ce document a été généré automatiquement le 1 novembre 2020.

Tous droits réservés 


\section{La narration au service de la politique monétaire : étude comparée du premier discours à Mansion House de deux gouverneurs de la Banque d'Angleterre}

Narration in the service of monetary policy: a comparative study of the maiden Mansion House speeches by two Governors of the Bank of England

\section{Laurence Harris}

1 La communication des banques centrales n'est pas spontanément associée au récit ou à la narration. Pourtant, dans leurs discours à l'occasion du banquet annuel donné par le Lord-maire de la City à Mansion House, il arrive que les gouverneurs de la Banque d'Angleterre empruntent au genre du récit. Ainsi, dès 1979, Gordon Richardson égrenait les mauvaises nouvelles (inflation en hausse, croissance en berne, avenir incertain) avant de conclure en ces termes: "I have told an unvarnished tale " (Richardson 1979).

2 L'emploi du terme tale est remarquable à plusieurs égards: c'est une première reconnaissance publique de l'importance du récit dans la présentation de la politique monétaire de la Banque d'Angleterre. S'il n'est pas aisé de trouver dans le reste du texte ${ }^{1}$ les fonctions structurales propres au conte établies par le sémiologue Vladimir Propp (1970), une autre déclaration corrobore la thèse de la mise en récit : « the moral of what I have been saying is plain» (idem). Le gouverneur exhorte ensuite industriels et ouvriers à prendre leurs responsabilités et à enrayer d'un commun accord la spirale des coûts. Cette reconnaissance sans doute involontaire que le discours de politique monétaire du gouverneur s'apparente à un conte préconisant une morale, ou un 
comportement approprié, confirme l'importance du récit dans la communication de la Banque centrale.

3 Dans cette étude, nous nous intéressons à deux autres gouverneurs de la Banque d'Angleterre, Mervyn King et Mark Carney, et, plus précisément, à leur premier discours à Mansion House. Ces discours ont été sélectionnés parmi soixante-et-onze allocutions prononcées à Mansion House depuis la nationalisation de la Banque en 1946 à nos jours car ils intègrent les structures narratives les plus abouties du corpus. En 2004, King évoque une marche du gouverneur à travers Londres inspirée de la fête irlandaise de Bloomsday; en 2014, Carney décrit un périple en canoë à travers les rapides canadiens dans l'esprit de la Conquête de l'Ouest. Après avoir précisé le cadre théorique et les choix méthodologiques qui ont servi de base à ce travail, nous nous attachons à analyser et à comparer les deux discours à travers leurs structures narratives afin de déterminer la visée de ces macro-récits. Nous affinons ensuite l'analyse en considérant les micro-récits enchâssés dans la trame discursive afin d'en comprendre la raison d'être et d'en tirer des enseignements. En adoptant cette démarche, nous cherchons à souligner l'intérêt que présente, en anglais de spécialité (ici, l'anglais de l'économie et de la finance), la prise en compte du contexte situationnel, culturel, temporel et discursif dans l'analyse des milieux spécialisés et de leurs acteurs.

\section{Cadre théorique et méthodologique}

4 Le cadre théorique de cette étude nécessite de convoquer les notions de genre, de rhétorique, de diachronie et de narration. Les deux discours étudiés sont extraits d'un corpus plus large d'allocutions des gouverneurs de la Banque d'Angleterre à Mansion House et s'inscrivent dans un genre particulier. Leur enjeu est de guider, de persuader ou de rassurer, d'où l'utilité d'envisager les aspects rhétoriques de ce type de discours. La perspective diachronique s'impose en raison des dix années qui séparent les deux discours. Enfin, la définition d'un sous-genre "premier discours à Mansion House " ouvre la possibilité d'étudier les aspects narratifs des textes.

5 Les discours des gouverneurs à Mansion House se distinguent d'autres déclarations publiques d'acteurs de la sphère financière. Ils s'apparentent à un domaine déjà abordé par Catherine Resche (2003), celui de la communication des banques centrales. Afin de circonscrire le genre auquel appartiennent ces discours, nous reprenons la définition qu'en propose John Swales (1990:58):

A genre comprises a class of communicative events, the members of which share some set of communicative purposes. These purposes are recognized by the expert members of the parent discourse community and thereby constitute the rationale for the genre. This rationale shapes the schematic structure of the discourse and influences and constrains choice of content and style.

6 Swales envisage le genre sous l'angle des objectifs communicationnels communs. La finalité commune aux discours de Mansion House est double. Elle rejoint d'une part les missions de la Banque centrale britannique, soit la stabilité monétaire du pays et la réduction du risque systémique. S'agissant par ailleurs des premiers discours à Mansion House, les nouveaux gouverneurs doivent aussi y présenter leur vision du rôle qui les attend et convaincre l'auditoire de leur compétence. La finalité des discours est donc également liée aux publics ciblés, qui jouent un rôle essentiel dans la compréhension et 
dans la production des discours. Au-delà des «banquiers et marchands de la City de Londres $»^{2}$ représentés au banquet par les invités du Lord-maire, les allocutions de Mansion House s'adressent à toutes les parties prenantes de la politique monétaire britannique; celles-ci incluent un public plus ou moins spécialisé d'investisseurs britanniques et s'étendent à la communauté financière mondiale des banques d'affaires et des fonds d'investissements. Pour autant, le lieu de l'événement reste l'une des constantes qui préside à l'élaboration des discours: ainsi le décorum unique de Mansion House (avec sa disposition particulière des convives, son code vestimentaire, son rituel de toasts portés à la famille royale et à la City) et le déroulement de l'événement (les médias sont présents mais silencieux, les discours n'étant pas suivis de conférences de presse) participent à l'homogénéité du genre. Enfin, il est nécessaire de prendre en compte l'identité sociale des partenaires langagiers. La fonction de gouverneur confère au sujet parlant un statut d'autorité qui légitime son droit à la parole. Chaque gouverneur reste toutefois libre de masquer ou de révéler certains traits de son identité personnelle et collective. Il faut un savant dosage à chaque gouverneur pour ne pas trop s'éloigner des codes permettant de reconnaître l'identité de la fonction tout en restant lui-même, avec son passé et sa personnalité, car l'individu ne saurait se réduire à une simple marionnette de l'institution ${ }^{3}$.

7 Ces contraintes ont une incidence sur le fond et sur la forme des discours. Leur influence s'observe, d'une part, sur l'homogénéité du domaine thématique (les allocutions à Mansion House entrent dans le cadre des annonces de politique monétaire de la Banque d'Angleterre, et sont répertoriées sur le site de la Banque parmi neuf catégories de publications comprenant notamment le rapport d'inflation trimestriel et le rapport biannuel de stabilité financière) et, d'autre part, sur certaines caractéristiques formelles relativement stabilisées des textes, tels les rites langagiers de politesse et les hommages au Lord-maire qui sont scrupuleusement observés. Cet ensemble de régularités traduit des comportements collectifs constitutifs d'un genre.

Une dimension rhétorique s'ajoute aux discours de Mansion House. Soucieux d'asseoir leur crédibilité afin de susciter la confiance de leurs publics et de donner la meilleure chance à leurs mesures d'être suivies d'effets, les gouverneurs ont amplement recours aux outils rhétoriques. Leur discours repose sur une rhétorique à la croisée des trois genres aristotéliciens (l'épidictique, le délibératif et le judiciaire), car il est l'occasion pour le locuteur de revenir sur le passé - afin de blâmer ou de louer, selon le bilan -, de commenter les indicateurs économiques du présent et de formuler ses projections sur l'avenir avec l'ambition d'y exercer une influence.

9 Notre choix des deux discours espacés de dix ans conduit naturellement à adopter une approche diachronique, qui présente aussi l'avantage de prendre en compte les contextes sociaux, économiques, politiques, sans parler de la personnalité des orateurs. L'approche diachronique nécessite un bref rappel des liens historiques qu'ont toujours entretenus économie et narration. Si la science économique et le roman sont nés en même temps (McCloskey 1992), le "tournant narratif » du discours économique remonte aux années 1990 (Salmon 2008) quand la première expérience de mise en récit dans la communication de la Banque mondiale a inauguré l'art du storytelling (Denning 2011) ; ayant gagné l'entreprise et le monde politique, celui-ci a connu des dérives - le titre de l'ouvrage de Christian Salmon sur ce sujet est éloquent: "Storytelling, la machine à fabriquer des histoires et à formater des esprits » (op. cit.). Les deux discours 
étudiés sont prononcés à une époque où l'usage de la narration dans le discours économique et politique s'accompagne aussi d'une plus grande méfiance.

Le cadre théorique éclaire la démarche méthodologique qui sous-tend l'étude. Elle s'effectue en deux étapes. La première étape consiste en une analyse comparée de la macro-structure des discours et vise à examiner, après un bref rappel des conditions d'énonciation, les références historiques, littéraires et humoristiques qui caractérisent l'incipit du récit et le format qu'adopte la narration centrale, afin de dégager les traits saillants de cette technique discursive. La deuxième phase de l'étude vise à isoler les récits annexes, moins développés que le récit central mais loin d'être secondaires. Leur fonctionnement sera décrypté afin de mesurer les enjeux qu'ils soulèvent. L'objectif de la démarche est de contribuer à la caractérisation de ce sous-genre particulier en analysant ses aspects narratifs.

\section{Macro-récit et finalité communicationnelle}

11 Par rapport à d'autres discours de gouverneurs prononcés dans d'autres circonstances et lieux, les deux discours dont il est question dans cette étude présentent la particularité d'intégrer des formes narratives. Leur trame narrative principale est explicite et sert de support au message du gouverneur dans le cadre des missions de la Banque. Une étude comparée de ces macro-récits - le terme-valise désigne ici à la fois l'outil narratif et les objectifs macroéconomiques de l'institution - met en lumière les parallèles et les points de divergence de ces deux discours.

\subsection{Situation d'énonciation}

Les auteurs de ces discours présentent des profils très différents. Britannique de cinquante-cinq ans et diplômé de Cambridge, Mervyn King a été promu en 2003 à la fonction de gouverneur après treize ans passés à la Banque en tant que directeur puis vice-gouverneur. Son discours de 2004 inaugure les dix années qu'il va passer à la tête de la Banque. Son successeur n'est pas un Britannique, une première dans l'histoire de la Banque: ex-gouverneur de la Banque du Canada et âgé de quarante-sept ans, le Canadien Mark Carney est diplômé de Harvard et d'Oxford, ainsi qu'ancien de Goldman Sachs. Son discours date de 2014.

Outre les locuteurs, les circonstances de l'énonciation diffèrent. Le discours de 2004 précède la crise financière de 2007-08 et se situe dans une période d'une quinzaine d'années caractérisée par une baisse de volatilité des marchés occidentaux. Le discours de 2004 fait d'ailleurs référence à un «âge d'or " pour la Banque. Le discours de 2014, en revanche, est prononcé au cours d'une période plus trouble. La crise financière s'est muée en une crise bancaire dont les répercussions sur l'économie réelle perdurent. La nomination surprise de Carney plutôt que celle du vice-gouverneur ou d'un candidat britannique en 2013 est annoncée sur fond de scandale du LIBOR ${ }^{4}$ (Hope 1992).

Ce sont des discours de début de mandat; il n'y a pas de bilan à défendre. Un hommage est rendu au prédécesseur comme la courtoisie et le rituel l'exigent lors d'un passage de témoin, mais le propos est tourné résolument vers l'avenir. Les questions macroéconomiques sont abordées, le double objectif de stabilité monétaire et financière de la Banque qui figure dans ses statuts depuis son indépendance est rappelé, quelques effets d'annonce sont ajoutés. Surtout, dans chaque discours, le 
locuteur se met en scène et décrit sa vision de la tâche à accomplir sous la forme d'un périple et à la première personne - un cas de mimésis très littéral, pour reprendre la formule de Paul Ricœur (2006).

Il est intéressant de noter qu'aucun des deux gouverneurs n'emploie les termes de « narrer », de « raconter » ou de « conte » contrairement au discours de 1979 de Gordon Richardson (op. cit.). King annonce qu'il va « décrire » son parcours, Carney précise qu'il établit une "analogie »: les termes choisis sont moins explicites. Si elle est devenue moins avouable du fait du discrédit frappant le storytelling, l'intention narrative reste intacte.

\subsection{Palimpseste et second degré}

Les incipits des deux discours puisent leurs sources dans des références littéraires ou anecdotiques qui apportent une épaisseur narrative au propos, tel un palimpseste, et se doublent d'une pirouette humoristique. Pour King, le centenaire de Ulysses (Joyce 2010 [1922]) et de la marche-hommage commémorative à travers Dublin le jour de la date anniversaire de l'opus, Bloomsday, lui fournit le prétexte d'inaugurer une «marche du gouverneur » à travers Londres :

You will all know that today is the centenary of "Bloomsday". Leopold Bloom's walk around Dublin, immortalised in James Joyce's Ulysses, was on 16th June 1904. Joyce himself wrote "I've put so many enigmas and puzzles [in the book] that it will keep the professors busy for centuries arguing over what I mean." Some might say that Joyce invented the language of central bankers. Tonight I want to describe a different walk - the Governor's walk. Initiated by Montagu Norman, from Notting Hill to the Bank of England, it is a walk through the British constitution. (King 2004)

Le ton est celui de l'humour, avec une allusion aux énigmes que Joyce a délibérément insérées dans son œuvre pour occuper les érudits pendant les siècles à venir : Joyce « a inventé le langage des banquiers centraux». King fait ainsi référence à la réticence passée des gouverneurs à s'exprimer clairement, eux qui sont prompts à brouiller les codes. Le nom du vénérable Montagu Norman ${ }^{5}$ est évoqué et fournit le signal du départ puisque le pèlerinage débute à partir de sa résidence de Notting Hill Gate, un procédé métonymique permettant de rappeler que l'histoire moderne de la Banque commence avec lui.

18 Carney évoque également l'incontournable Norman, mais sans la même déférence; c'est surtout pour rappeler avec ironie le talent qu'avait ce dernier à se soustraire aux décisions difficiles, par exemple en 1931 lorsqu'il part en villégiature au Québec pour des raisons de santé alors que la conjoncture économique britannique se dégrade :

One has only to look back to 1931 when Britain's economic prospects were strained by high unemployment, a large budget deficit and a deteriorating balance of payments. In the ensuing crisis the government of the day resigned and sterling was forced off the gold standard. And the Governor? With the uncanny foresight of a central banker, Montagu Norman had already left the scene 'to get a bit of rest [in Quebec]', he said.

However tempting, I will lean on Canada not for restoration but for a nautical analogy to describe how we can address the challenges we now face. (Carney 2014)

Cette référence aux années trente souligne les dangers qui guettent un pays dont la balance des paiements est déficitaire et l'allusion au Québec fournit le lien nécessaire au départ du périple canadien. Toutefois Carney s'attarde d'abord sur Norman, qui a su 
faire preuve d'une « clairvoyance singulière » en disparaissant au moment opportun, ce qui lui permet une pique à l'encontre de sa propre fonction.

Les gouverneurs sont inintelligibles, se moque King en 2004 ; les gouverneurs sont des dilettantes, renchérit Carney en 2014 - sans doute veut-il aussi souligner leur perspicacité. Si l'esprit de Montagu Norman est invoqué pour des motifs différents, la technique de l'autodépréciation vise dans les deux cas à créer une connivence avec l'auditoire qui est invité à suivre ou à embarquer - le voyage peut commencer.

\subsection{De Leopold Bloom à Davy Crockett}

La marche tranquille du gouverneur conduit King à travers les parcs londoniens, lui laissant le loisir d'admirer les bâtisses royales ainsi que les symboles des branches de la constitution (exécutif, législatif et judiciaire) :

Starting in the "dignified" branch of the constitution, to use Walter Bagehot's word, near Kensington Palace it continues through the Royal Parks towards the

"efficient" part of the constitution: the Executive. (King op. cit.)

Chaque station est l'occasion d'un commentaire, soit par le biais d'une citation d'un illustre prédécesseur - Walter Bagehot, le gouverneur Cobbold, le vice-gouverneur Harvey - soit en aparté. Faisant une halte sous les fenêtres du Chancelier à Whitehall, il se permet une observation sur les dépenses de l'État qu'il juge excessives : «I look up at the Chancellor's window and see those famous golden scales [...]. Recently they have tilted more and more towards the spending side » (idem).

King saisit cette occasion pour rappeler les devoirs du gouverneur: conseiller l'exécutif, mais aussi rendre des comptes au Parlement - il évoque ainsi ses quatre convocations en un an devant la Commission des finances. Cette marche-hommage s'inscrit dans une verticalité qui appuie sa déférence vis-à-vis du pouvoir. King gravit l'escalier le menant au ministère des finances - les Clive Steps célèbrent en filigrane la grandeur de l'Empire britannique : «Up the Clive Steps, and navigating carefully between the Foreign Office and the Treasury [...]». Sous les fenêtres du Chancelier, il lève les yeux ( «I look up ») vers la balance gravée sur le fronton du Trésor, symbole d'équité (id.).

Il lui reste une ultime épreuve avant d'atteindre les portes de la Banque, franchir le Millenium Bridge :

The Governor's walk now crosses Westminster Bridge, continues down the South

Bank and over to that other monument of stability, The Millenium Bridge.

I enter the City [...] and arrive in the calm atmosphere of the Bank of England. (id.)

Le pont a conservé son surnom de "wobbly bridge » dont l'évocation ajoute une touche de dérision par effet de décalage visant à atténuer la pompe du propos, mais rappelle également les incertitudes qui guettent la fonction de gouverneur.

La bonhomie teintée de solennité de ce premier parcours contraste avec le périple que relate Mark Carney. Son discours de 2014 détourne d'autres codes, ceux de la Conquête de l'Ouest. Une analogie nautique structure le propos et permet de filer deux métaphores, celle de l'eau et celle de la navigation.

C. Resche (2013 : 162-168) a souligné l'omniprésence de la métaphore liquide dans le discours économique, en particulier dans la production textuelle britannique - la Grande-Bretagne est une île, sa flotte lui permit de dominer les mers. Carney s'approprie cette métaphore souche. Il n'est pas du sérail du fait de ses origines 
canadiennes, mais il légitime sa nomination en s'inscrivant dans une longue lignée de gouverneurs britanniques qui ont usé et abusé de cette veine métaphorique.

Son embarcation de choix est le fidèle canoë, modeste mais maniable dans les rapides canadiens dont les eaux sont traitresses : « a craft that can navigate the most rapid and treacherous waters» (Carney op.cit.). Ces dangers appellent des qualités d'habileté et d'adaptation qui contrastent avec l'apologie de la bonhomie et de la lenteur chez King.

Si le canoë définit la fonction, la métonymie se poursuit avec une autre embarcation évoquée par Carney, le yacht majestueux qu'affectionnait son prédécesseur: "rather than appeal to the stately Duchess of York on which my predecessor sailed» (idem). Doit-on déceler sous l'hommage une allusion déguisée au bilan flatteur dont Carney se targue à la tête de la Banque du Canada, son savoir-faire au cœur de la tourmente de 2008 ayant épargné à son pays la crise des subprimes, contrairement à son prédécesseur qui fut vilipendé dans la presse pour son manque de réactivité dans le sauvetage de Northern Rock?

Quoi qu'il en soit, c'est mué en trappeur canadien que le nouveau gouverneur s'élance dans les rapides. Chaque virage et coup de rame est l'occasion d'évoquer un nouveau volet de sa politique monétaire.

31 Le rédacteur du discours fait le choix de mettre à nu le procédé rhétorique. Pas de couleur locale ou d'élaboration d'une intrigue; il n'est pas précisé non plus si la navigation est ascendante ou descendante: deux fois plus long que celui de 2004, ce discours, plus technique sur les questions de finance, est également émaillé de lieux communs, de proverbes attendus et d'idiomes éculés sur le thème de l'eau. Ces topoi qui ponctuent le discours permettent des pauses en suscitant un sourire, mais illustrent aussi des notions que le gouverneur met en avant. Ainsi l'insistance sur la synchronisation des coups de rames permet une transition vers la nécessité des efforts coordonnés des autorités fiscales, monétaires et prudentielles.

32 "This may be a case of still waters running deep - often the most dangerous time on the river" (id.) : ce proverbe est l'occasion d'évoquer les changements nécessaires au sein du FCA ${ }^{6}$ afin de lui permettre d'accroître sa vigilance dans la surveillance des marchés. De même, "When you hear the thunder of the falls, it is wise to get off the river" (id.) est la reformulation d'un couplet sur l'anticipation du risque dans le marché immobilier - la remarque est d'ailleurs introduite par " in other words ».

Quant à la tournure très familière "we are not up the proverbial creek without a paddle » (id.), elle permet d'aborder les moyens dont dispose la Banque, hormis la hausse des taux d'intérêt, pour enrayer les effets néfastes de la dette des ménages. Cette dernière saillie fait presque figure de provocation dans le contexte un peu empesé et révérencieux qui caractérise le banquet de Mansion House.

Carney imprime son style. Il conclut l'allocution sur un point de divergence supplémentaire avec son prédécesseur. Son périple n’a pas de fin : « unlike a canoe trip, the quest for economic balance never ends» (id.). L'analogie avec le parcours en canoë atteint son terme et, une fois de plus, ce point est exprimé de façon explicite.

L'exposé se conclut sur un des mots clés du discours: "with economies and markets always moving between equilibria, it is the journey that matters" (id.). La mission du gouverneur est la recherche des équilibres dans un monde en mouvement. Cette nouvelle normalité ou anormalité (Bremmer \& Roubini 2013) signe la fin de la recherche d'une stabilité illusoire qui n'est peut-être pas souhaitable. On peut voir dans 
le parcours de ce Davy Crockett s'aventurant aux frontières de la narration une parenté avec le retour à l'équilibre que le sémiologue Todorov (1973) met en évidence dans la structure commune des histoires et que l'on retrouve également chez Saussure (Resche op. cit. : 267).

Une lecture comparée de ces deux premiers discours prononcés à dix ans d'intervalle permet de formuler quelques remarques sur leurs aspects narratifs. Les macro-récits épousent une structure semblable : l'amorce puise ses sources dans l'histoire commune de la Banque en célébrant le même héros, mais également dans les références littéraires ou culturelles personnelles de chaque locuteur. Le récit proprement dit est celui d'un parcours dont les modalités - direction, locomotion, cadence, but traduisent une vision de la fonction de gouverneur inspirée des circonstances de l'énonciation (les perspectives de la Grande-Bretagne diffèrent avant et après la crise de 2008) et de la personnalité de l'orateur. Carney, dont le ton est plus détaché et le récit moins personnalisé que ceux de son prédécesseur, s'approprie en 2014 les métaphores et métonymies qui accompagnent le cheminement de King en 2004. La finalité communicationnelle du discours explique la recherche commune de l'équilibre et de la stabilité dans un monde où les facteurs de contingence externe échappent au contrôle du gouverneur.

Les récits annexes qui viennent se greffer sur la trame narrative principale soulèvent de nouveaux enjeux.

\section{Micro-récits et enjeux rhétoriques}

Plusieurs micro-récits qui empruntent à l'allégorie, à l'anecdote et à la mythologie ou qui font écho à d'autres discours étoffent la trame narrative principale du périple et l'enrichissent d'une visée d'ordre rhétorique. C'est le cas de la correspondance que l'on peut établir entre les récits des parcours et le graphisme des prévisions chiffrées de la Banque d'Angleterre qui accompagnent le rapport d'inflation trimestriel, les fan charts ou projections en éventail.

\section{1. «Allégories économétriques » : faire participer le public à la modélisation de l'économie}

La Banque d'Angleterre est pionnière dans la conception du fan chart, mode de représentation d'une distribution de probabilité provisionnelle ${ }^{7}$. Il s'agit d'une évaluation par les membres du MPC (Monetary Committee Policy), comité de décideurs dont le gouverneur fait partie au sein de la Banque, des routes possibles que pourrait emprunter une variable macroéconomique, par exemple l'inflation, sous la forme d'un tracé de bandes de couleurs plus ou moins foncées selon la solidité des convictions du groupe. Or la genèse de ces fan charts repose sur le récit et sur le cheminement. Les données qui permettent ces estimations sont des conversations recueillies sur le terrain par les douze agences régionales de la Banque auprès d'un large échantillon d'acteurs économiques. Depuis septembre $1997^{8}$, un « réseau " (« the network») de 7000 contacts issus des secteurs public et privé est régulièrement sollicité : environ 700 d'entre eux sont consultés tous les mois sur des sujets variés - une liste de "hot topics" est préétablie par la Banque; les membres du MPC accompagnent par ailleurs les agents dans leurs visites environ six fois par an. David Blanchflower, ancien membre du MPC, 
nomme cette collecte de récits individuels "the economics of walking about " (Holmes 2014 : 159-161). Les rapports compilés d'après ces échanges sont étudiés par le MPC avant le début de leurs délibérations sur les taux d'intérêts, et même s'ils demeurent confidentiels, ces récits finissent par filtrer dans les comptes rendus trimestriels des réunions du MPC. La publication des procès-verbaux du MPC n'a pas pour objectif une plus grande transparence de la part de la Banque, bien que celle-ci affirme le contraire (Warsh 2014) : ces « allégories économétriques » (Blinder \& Ricardo 2005) visent avant tout à orienter les attentes des consommateurs jusqu'à les faire participer à la modélisation de l'économie. Ces derniers sont les protagonistes d'une histoire narrée par la Banque centrale, histoire qu'elle a rédigée au préalable et de concert avec les marchés.

Ainsi, la communication multiforme de la Banque d'Angleterre (rapport d'inflation, bulletin trimestriel, etc., et, bien sûr, discours de Mansion House) ne constitue pas un ensemble de commentaires, d'analyses ou d'interprétations de la situation économique: elle est la politique économique. En d'autres termes, la Banque mise en partie sur la performativité du langage pour mener à bien ses objectifs macroéconomiques ${ }^{9}$. D'après D. R. Holmes (op. cit. : 1), les sciences prévisionnelles ont cédé le pas à l'ethnographie et à l'anthropologie par le biais de pratiques communicatives complexes intégrant entre autres le récit et visant à établir la confiance dans la devise nationale. Selon lui, la notion de "fiat money» simplement fondée sur la confiance portée à un gouvernement ne suffit plus; il note l'emploi par Mervyn King, sans doute par inadvertance, de l'expression plus juste de «public currency ». La collaboration entre les décideurs et les acteurs de la sphère financière est la base du système.

\subsection{L'effet Wimbledon : accepter la mondialisation}

41 La narration du périple annonce la vision qu'a le nouveau gouverneur de ses fonctions et la cadence qu'il souhaite imprimer à ses réformes. Le pas nonchalant et mesuré de King révèle une vision teintée de nostalgie et de patriotisme. Il est rattrapé par la cadence accélérée de son successeur, qui bouscule les habitudes et les traditions. Le discours de Carney est plus long, plus technique, la version écrite comprend de nombreuses notes de bas de page ainsi qu'une bibliographie et signale sa volonté d'imposer plus de professionnalisme à cet exercice de communication.

King se félicite en 2004 de l'effet Wimbledon, référence à un grand tournoi sportif britannique où les meilleurs sont invités, mais qui ne consacre pas nécessairement un champion national. Il pressent l'inéluctable ouverture de la Grande-Bretagne à la mondialisation, déjà observée dans d'autres sphères de l'industrie britannique :

Since Big Bang in 1986, the City has changed beyond all recognition. The so-called "Wimbledonisation" of the City - hosting a successful tournament where most of the winners come from overseas - has proceeded apace. Some have blamed the Bank, among others, for failing to engineer the promotion of more British institutions to the top ranks of global financial institutions. But in fact there are now some home players in the top ten in the world. And there is little evidence that it makes sense for the public sector to try to identify national champions, as opposed to creating an environment which encourages innovation and provides first-rate infrastructure. (King op. cit.) 
Ce phénomène de "Wimbledonisation » annoncé dans le discours de King se réalise au sommet de la Banque avec la nomination de Carney, un choix qui ne fait pas l'unanimité en 2013. La Banque d'Angleterre à cette époque est pour Carney non pas l'apogée de sa carrière, ni une fin en soi, mais plutôt une étape : il annonce qu'il ne servira que cinq années de son mandat de huit ans à la tête de la Banque avant de revenir sur cette décision fin 2015, ses ambitions politiques étant contrariées par la victoire de Justin Trudeau au poste de Premier Ministre du Canada. Autre conséquence plus anecdotique de l'effet Wimbledon, Carney met un terme, un an après sa nomination, au match de cricket annuel organisé à l'occasion d'une fête réservée aux employés de la Banque. Simple fait divers au regard de la politique monétaire d'un pays $^{10}$, c'est pourtant dans cette même tradition que s'inscrit le rituel de Mansion House. Le patriotisme dont King fait preuve dans son discours semble presque désuet dix ans plus tard.

\subsection{Politique monétaire « odysséenne » ou « delphique » : piloter les anticipations}

Carney vante dans son discours les mérites d'un nouvel outil, "Forward Guidance »" Concrètement, le gouverneur pilote les anticipations des acteurs financiers en s'engageant à ne pas relever les taux d'intérêt avant que le taux de chômage soit inférieur à $7 \%$. C'est en tout cas ce qu'il a annoncé lors de sa nomination dès 2013, une option audacieuse qualifiée d'odysséenne dans le jargon monétaire - terme intéressant pour ses connotations narratives et signifiant que la banque centrale se lie les mains à l'avance de façon à ne pas pouvoir dévier du cap sur lequel elle s'est engagée, comme l'Ulysse d'Homère se lie au mât de son navire pour résister aux tentations des sirènes.

Or, le chômage a chuté de façon inattendue en dessous de 7 \% en quelques mois (ce qui constitue a priori une bonne nouvelle), mais les conditions générales de l'emploi ne permettant pas encore une hausse des taux d'intérêt, le gouverneur s'est trouvé embarrassé. Cette chute inattendue ne devait rien à sa politique monétaire - dont les effets se font normalement sentir au bout de quinze à dix-huit mois - et l'a obligé à des rectificatifs, à des annonces contradictoires et à une transition discrète vers la seconde option, moins contraignante, plus consensuelle, qualifiée de delphique, d'après l'oracle de Delphes où la Pythie transmettait dans un langage énigmatique les messages d'Apollon sur l'avenir. La Banque centrale se contente alors d'indiquer un chemin qu'elle se réserve le droit de revisiter. Le risque est que ce second type de forward guidance soit perçu comme un non-événement. Les médias se sont d'ailleurs empressés de renommer le procédé «fuzzy guidance» (Elliott 2014). Ensuite les marchés, sans direction claire, en viennent à spéculer sur la date d'une éventuelle hausse - pas avant la fin de son mandat, disent certains. Servir un mandat entier sans modifier sa politique monétaire, seul le gouverneur Catto s'y est risqué dans les années 40 , et ce n'est certainement pas un chapitre de son parcours ou de l'histoire de la Banque que Carney souhaite écrire.

\subsection{Discours d'autorité : valoriser son ethos}

Un dernier enjeu explique le recours aux micro-récits : le nouveau gouverneur saisit cette occasion de valoriser son ethos afin d'assurer sa crédibilité. Le discours n'est pas 
seulement destiné à être compris, c'est aussi un signe d'autorité destiné à être cru ou auquel on obéit (Bourdieu 1982:105). Une difficulté majeure dans cette entreprise réside dans la nécessité de ménager l'autre orateur prestigieux de la soirée, le Chancelier de l'Échiquier, car ce dernier s'exprime également devant la nation lors du banquet de Mansion House. Il intervient juste avant le gouverneur et ses propos sont tout autant - sinon davantage - suivis et décryptés. L'exercice est d'autant plus délicat qu'il s'agit pour le gouverneur de ne pas voler la vedette au Chancelier, ni de lui faire de l'ombre, mais au contraire d'appuyer son propos, de lui faire écho, car le gouverneur occupe l'un des postes les plus puissants du Royaume sans y avoir été élu : il y est nommé par le Chancelier.

La frontière entre complicité et déférence est ténue. Si l'on compare les discours des deux gouverneurs avec les allocutions des Chanceliers présents les mêmes soirs, Gordon Brown (Brown 2004) et George Osborne (Osborne 2014) ${ }^{12}$, il est intéressant de noter que les Chanceliers citent tous deux Winston Churchill, qui a été Chancelier de l'Échiquier, de même que les deux gouverneurs évoquent de leur côté Montagu Norman - à chaque fonction son héros. Certaines stratégies émergent dans le rapport de politesse, l'écho des thèmes et la symétrie du propos, allant jusqu'à la flatterie. Des écueils guettent les nouveaux gouverneurs : King se risque à critiquer dans son discours les dépenses excessives de Gordon Brown. Les immixtions de Carney dans la sphère politique constituent également un exercice périlleux : est-il trop proche de l'exécutif lorsqu'il s'implique en 2014 dans le débat sur l'indépendance de l'Écosse trois semaines avant le référendum? Ou lorsqu'il est sommé par le Parlement britannique de réitérer la neutralité de la Banque sur la question du Brexit (Demianyk 2016)?

Cette deuxième grille de lecture des discours a permis de déceler une variété de microrécits, enchâssés dans la trame discursive, qui étoffent la portée des discours d'une visée rhétorique. Le gouverneur a recours à ces allégories économétriques, anecdotes ou références mythologiques afin de gagner la confiance du public, condition préalable au succès de sa politique monétaire. Il s'assure la participation des acteurs économiques aux objectifs de stabilité de la Banque, diffuse un message rassurant sur les incertitudes liées à la mondialisation et pilote les anticipations des consommateurs. La symétrie entre ses micro-récits et ceux du Chancelier relève du calcul politique, cette stratégie permettant au gouverneur de s'assurer le soutien de l'exécutif et de légitimer son autorité.

Dans les discours qui suivent ceux des soirées inaugurales de 2004 et 2014, les deux gouverneurs adoptent de nouveaux procédés communicationnels et rhétoriques qui semblent les éloigner de la narration. Ils ont alors un bilan à défendre, des justifications à fournir. Les mauvaises nouvelles s'accommoderaient-elles mal d'une mise en récit aux ficelles trop visibles?

\section{Conclusion}

L'étude comparée des allocutions de 2004 et de 2014 à Mansion House permet une première caractérisation du sous-genre des premiers discours sur une période récente. La narration y occupe une place centrale. Le mandat du nouveau gouverneur est annoncé sous la forme d'un macro-récit dont les points essentiels dessinent les jalons d'un parcours plus ou moins mouvementé selon les circonstances et le locuteur. Les micro-récits qui se greffent sur la trame centrale ajoutent à la visée 
communicationnelle une visée rhétorique : le gouverneur formule sa vision des défis à relever en misant sur la performativité du langage.

Ce format narratif, inédit avant 2004, remplit deux fonctions. Il permet au gouverneur d'asseoir son autorité au sein de la City. La narration devient une version moderne des «Governor's eyebrows » - allusion au froncement de sourcils du gouverneur qui suffisait à mettre au pas les marchés financiers à une époque où le mutisme était de mise à la Banque d'Angleterre. La deuxième fonction que remplit la mise en récit est la captation de l'auditoire : la parole du gouverneur doit rester audible aux communautés ciblées, spécialisées ou non.

On ne peut certes conclure de façon définitive sur les traits narratifs de ce genre particulier à partir de deux discours seulement, mais cette contribution pourra nourrir une étude consacrée à un corpus beaucoup plus large et portant sur la récurrence des métaphores, la polyphonie, la poésie, l'humour ou le mythe ${ }^{13}$ afin de vérifier si ces procédés tissent d'autres trames qui assurent cohérence et cohésion au rituel de Mansion House d'une année à l'autre, reprenant peut-être le fil d'un récit ininterrompu.

\section{BIBLIOGRAPHIE}

\section{Sources primaires}

BROWN, Gordon. 2004. " Speech given by the Chancellor at the Mansion House, London ", The Guardian, 17 June, consulté le 14/06/2016 <http://www.theguardian.com/politics/ 2004/jun/17/ economy.uk>.

CARNEY, Mark. 2014. « Speech given by the Governor at the Lord Mayor's Banquet for Bankers and Merchants of the City of London at the Mansion House, London, on 12 June 2014 », consulté le 14/06/2016 <http://www.bankofengland.co.uk/publications/Pages/speeches/ 2014/736.aspx>.

KING, Mervyn. 2004. «Speech Given by the Governor at the Lord Mayor's Banquet for Bankers and Merchants of the City of London at the Mansion House, London, on 16 June 2004 ", consulté le 14/06/2016 <http://www.bankofengland.co.uk/archive/Documents/historicpubs/ speeches/ 2004/speech222.pdf>.

OSBORNE, George. 2014. « Mansion House 2014: Speech by the Chancellor of the Exchequer », HM Treasury, consulté le 14/06/2016 <https ://www.gov.uk/government/speeches/mansionhouse-2014-speech-by-the-chancellor-of-the-exchequer>.

RICHARDSON, Gordon. 1979. « Speech Given by the Governor at the Lord Mayor's Banquet for Bankers and Merchants of the City of London at the Mansion House, London, on 18 October 1979 ", consulté le 14/06/2016 <http://www.bankofengland.co.uk/archive/Documents/ historicpubs/qb/1979/qb79q4407415.pdf>.

\section{Sources secondaires}

BLINDER, Alan S. \& Reis RICARDO. 2005. «The Greenspan Standard. Paper presented at the Federal Reserve Bank of Kansas City symposium », Jackson Hole, Wyoming, 25-27 August. 
BOURDIEU, Pierre. 1982. Ce que parler veut dire - l'économie des échanges linguistiques. Paris : Fayard.

BREMMER, Ian \& Nouriel ROUBINI. 2013. «Ian Bremmer and Nouriel Roubini unveil the new

abnormal », Institutional Investor, 17 June, consulté le 14/06/2016. <http://

www.institutionalinvestor.com/Article/3218470/Ian-Bremmer-and-Nouriel-Roubini-Unveil-theNew-Abnormal.html>.

CHARAUDEAU, Patrick (dir.). 2009. Identités sociales et discursives du sujet parlant. Paris : L'Harmattan.

EMIANYK, Graeme. 2016. « Mark Carney Condemned by Jacob Rees-Mogg for 'Brexit'

'Propaganda », The Huffington Post, 24 May, consulté le 14/06/2016. <http://

www.huffingtonpost.co.uk/entry/mark-carney-jacob-rees-mogg-

brexit_uk_57444a41e4b00006e9af2e90>.

DENNING, Stephen. 2011. The Leader's Guide to Storytelling: Mastering the Art and Discipline of Business Narrative. San Francisco : John Wiley \& Sons.

ELLIOTT, Larry. 2014. « Forward Guidance Version 2: Will the Public Believe it? », The Guardian, 12 February, consulté le 14/06/2016. <https://www.theguardian.com/business/economics-blog/ 2014/feb/12/bank-of-england-inflation-report-forward-guidances.

« Historical Fan Chart Data ». 2016. Bank of England, consulté le 14/06/2016. <http:// www.bankofengland.co.uk/archive/Documents/historicpubs/qb/1979/qb79q4407415.pdf>. HOLMES, Douglas R. 2014. Economy of Words: Communicative Imperatives in Central Banks. Chicago : Chicago University Press.

HOPE, Christopher. 1992. « Libor Scandal Taints Favourites in Running for Bank of England's Top Job ». The Telegraph 17 August 1992, consulté le 14 juin 2014. <http://www.telegraph.co.uk/ finance/9483898/Appointment-of-new-Bank-of-England-Governor-could-be-delayed.html>. JOYCE, James. 2010 [1922]. Ulysses. Ware : Wordsworth Editions.

KYNASTON, David. 2012. City of London. The History. Londres : Vintage.

LEBOR, Adam. 2014. Tower of Basel. New York : PublicAffairs.

MCCLOSKEY, Deirdre N. 1992 [1990]. If You're so Smart - The Narrative of Economic Expertise. Chicago : University of Chicago Press.

PROPP, Vladimir. 1970. Morphologie du conte. Paris : Seuil

RESCHE, Catherine. 2003. « Décryptage d'un genre particulier : les communiqués de presse de la Banque Centrale américaine ». ASp 39-40, 21-35.

RESCHE, Catherine. 2013. Economic Terms and Beyond: Capitalising on the Wealth of Notions: How Researchers in Specialised Varieties of English Can Benefit from Focusing on Terms. Berne : Peter Lang.

RICEUR, Paul. 2006 [1983]. Temps et récit - Tome 1 : l'intrigue et le récit historique. Paris : Seuil. ROWLEY, Emma \& Graham Ruddick. 2011. « Bank of England Governor Mervyn King Spends the Day Watching England Cricket Team at Lord's », The Telegraph, 22 July, Consulté le 14/06/2011. $<$ http ://www.telegraph.co.uk/finance/economics/8653299/Bank-of-England-Governor-MervynKing-spends-the-day-watching-England-cricket-team-at-Lords.html>.

SALMON, Christian. 2008 [2007]. Storytelling, la machine à fabriquer des histoires et à formater les esprits. Paris : La Découverte Poche. 
SWALES, John M. 1990. Genre Analysis: English in Academic and Research Settings. Cambridge : Cambridge University Press.

The Times. 1877. « Banquets ». Times Newspaper Limited. Issue 28,926. 25 April, 10.

TODOROV, Tzvetan. 1973. Qu'est-ce que le structuralisme? Poétique, tome 2. Paris : Seuil.

WARSH, Kevin. 2014. « Transparency and the Bank of England's Monetary Policy Committee », Review. Bank of England, Consulté le 14/06/2016. <http ://www.bankofengland.co.uk/ publications/Documents/news/2014/warsh.pdf>

YELLEN, Janet L. 2013. « Communication in Monetary Policy », Remarks by the Vice Chair, Board of Governors of the Federal Reserve System at the Society of American Business Editors and Writers, 5th Anniversary Conference, Washington D.C., 4 April.

\section{NOTES}

1. Le conteur a prévenu son auditoire, cette histoire est narrée «sans fard » (« unvarnished ») : il n'y a ni héros ni intrigue à proprement parler.

2. La formule «Bankers and Merchants of the City " figure dans la plus ancienne référence au banquet de Mansion House retrouvée dans la presse (The Times 1877) ; elle est reprise aujourd'hui encore dans les intitulés complets des discours («Speech Given by the Governor at the Lord Mayor's Banquet for Bankers and Merchants of the City of London at the Mansion House, London »).

3. Charaudeau (2009: 15) décrit ce jeu de va-et-vient entre l'identité sociale et l'identité discursive comme un « jeu de miroir fondateur de l'activité langagière ».

4. Le LIBOR (London Interbank Offered Rate) est un taux d'intérêt de référence interbancaire établi à Londres.

5. Montagu Norman, gouverneur légendaire resté vingt-quatre ans (de 1920 à 1944) aux commandes de l'institution en raison des circonstances exceptionnelles de la Seconde Guerre mondiale, préconisait le silence en toute circonstance : sa devise était «never explain, never excuse ». Il entretenait la culture du secret qu'il estimait nécessaire à l'intérêt de la Banque (Lebor 2014). Son influence pèse sur les mandats des neuf gouverneurs qui lui ont succédé jusqu'à aujourd'hui, période au cours de laquelle la Banque est passée de la culture du mutisme à celle de l'ouverture.

6. Le Financial Conduct Authority est l'Autorité britannique des marchés financiers.

7. De nombreux documents sont consacrés à l'élaboration de ces fan charts sur le site de la Banque d'Angleterre. (« Historical Fan Chart Data » 2016).

8. ... lorsque le ciblage d'inflation est devenu le dispositif de politique monétaire officiel de la Banque d'Angleterre.

9. Comme l'admet la présidente de la Réserve fédérale, "Sometimes the explanation is the policy " (Yellen 2013).

10. Féru de cricket, King essuie les critiques de la presse lorsqu'il s'offre des escapades à Lord's en pleine crise de l'eurozone (Rowley \& Ruddick 2011).

11. Cet outil de politique monétaire utilisé par les banques centrales consiste à annoncer une trajectoire pour les des taux d'intérêt sur le moyen ou long terme dans le but d'influencer durablement les anticipations du public quant à l'évolution des prix.

12. Un mandat de gouverneur dépasse généralement en durée celui d'un Chancelier, mais en 2004 et 2014, King et Carney retrouvent au Banquet de Mansion House les Chanceliers qui les ont nommés à leur poste, respectivement Brown et Osborne.

13. C'est l'objet de nos travaux de recherche dans le cadre d'une thèse en cours portant sur le discours annuel du gouverneur de la Banque d'Angleterre à Mansion House et couvrant une 
période de soixante-dix ans (1946-2016). Cette contribution à l'étude des discours spécialisés aborde l'évolution dans la forme et dans le contenu des allocutions en parallèle avec l'internationalisation de la City, l'émergence des médias de masse et l'ouverture de la Banque d'Angleterre à une plus grande transparence.

\section{RÉSUMÉS}

Cette étude propose d'explorer les frontières narratives en anglais de l'économie et de la finance à partir de deux discours de gouverneurs de la Banque d'Angleterre extraits d'un corpus de soixante-et-onze allocutions prononcées depuis la nationalisation de la Banque en 1946. L'étude se présente en trois parties. La première partie aborde le cadre théorique et méthodologique de l'étude. La deuxième partie mène une lecture comparée des deux discours du corpus intégrant les structures narratives les plus abouties et s'interroge sur la visée de ces macro-récits. La troisième partie examine la signification des micro-récits enchâssés dans la trame discursive et les enjeux qu'ils soulèvent. L'objectif est de mettre au jour les ressorts narratifs auxquels les gouvernements ont recours afin de capter l'attention et d'obtenir l'adhésion de plusieurs publics, tout en parvenant à inspirer confiance, à rassurer et à guider face à de multiples incertitudes.

This study aims to explore the narrative margins in English for economics and finance by reference to two speeches by Governors of the Bank of England extracted from a corpus of seventy-one speeches delivered since the nationalisation of the Bank in 1946. The study is in three parts. The first part broaches the theoretical and methodological framework which underpins the project. The second part takes the form of a comparative reading of the two speeches in the corpus integrating the most developed narrative structures and addresses the purpose of these macro-tales. The third part examines the meaning of micro-tales embedded in the discursive frame and the stakes they raise. The goal is to demonstrate how gaining the attention of a varied audience, inspiring trust and leading the way in the face of uncertainty are challenges Governors meet using narrative techniques, amongst others.

\section{INDEX}

Keywords : Bank of England, econometric allegory, macro-tale, micro-tale, narration, rhetoric Mots-clés : allégorie économétrique, Banque d'Angleterre, macro-récit, micro-récit, narration, rhétorique

\section{AUTEUR}

\section{LAURENCE HARRIS}

Agrégée d'anglais, Laurence Harris enseigne l'anglais de l'économie et la civilisation britannique à l'Université Paris Nanterre. Ses recherches en cours portent sur la communication de la Banque d'Angleterre. Elle a publié en 2013 « The BBC in its own words » dans Expériences de guerre (Dickason [dir.], coll. Politeia, Mare \& Martin). laurence.harris@u-paris10.fr 